\title{
Use of Meteorological Information for Condition Monitoring of Transmission Lines in Plateau Areas
}

\author{
Xiaomin MA \\ State Grid Sichuan Electric \\ Power Research Institute \\ Chengdu, China \\ 373003345@qq.com
}

\author{
Songhai FAN \\ State Grid Sichuan Electric \\ Power Research Institute \\ Chengdu, China \\ 787855837@qq.com
}

\author{
Wei LI \\ Guizhou Institute of \\ Technology \\ Guiyang, China \\ 595896359@qq.com
}

\begin{abstract}
Keywords: plateau areas, condition monitoring, meteorological information, environmental meteorology classification
\end{abstract}

\begin{abstract}
Environmental meteorological conditions are important factors that affect the operation of transmission lines, and further decide the reliability of the operation condition monitoring of transmission lines in plateau areas, especially in the long-term lasting power supply of condition monitoring devices, therefore the accurate acquisition of meteorological information in this area and making full use of it are particularly critical. In this paper it was proposed to the key environmental meteorological impact factors that affect the selection of power supply for the condition monitoring of transmission lines in plateau areas and the clustering algorithm was used to realize the environmental meteorology classification in plateau areas, which could provide the basis information for the power supply selection of condition monitoring devices for transmission line in plateau area. The comparative analysis showed that the classification results were reasonable and effective.
\end{abstract}

\section{Introduction}

In recent years, condition monitoring devices have been more and more widely used to the transmission lines in plateau area, especially in high-altitude and harsh environment areas with complex operating conditions and difficult operation and maintenance management [1-3]. On September 10, 2019, the 26 iron towers of Changji-Guquan UHV transmission line with a length of about 13 kilometers $\pm 1100 \mathrm{kV}$ completed the status monitoring information back to the transmission line online monitoring system, achieved the online monitoring data transmission of the transmission line of the first UHV DC highest voltage level in in plateau area in China and filled the gap that the online monitoring signal of the UHV line in plateau area was not be transmitted in the past, which is as shown in Fig.1.

Currently, three technical methods are commonly applied to the power supply of transmission line condition monitoring devices [4], including solar energy + battery, wind energy + battery, solar energy + wind energy + battery. Of course, there are also a few adopting induction energy supply ways on the transmission line [5].

According to statistics on site from power department, the power failures of condition monitoring devices accounted to more than $70 \%$ of the total failures in power transmission lines in plateau areas, most of which were caused by environmental meteorological conditions. The power supply problem 
has become the bottleneck of condition monitoring device of the transmission line in high altitude areas, especially in the power supply selection of these monitoring device. Therefore, the meteorological influencing factors will be studied in this paper to improve the environmental adaptability of the power supply of the condition monitoring device and ensure the lasting and reliable operation of this monitoring device in plateau areas, which is of great significance.

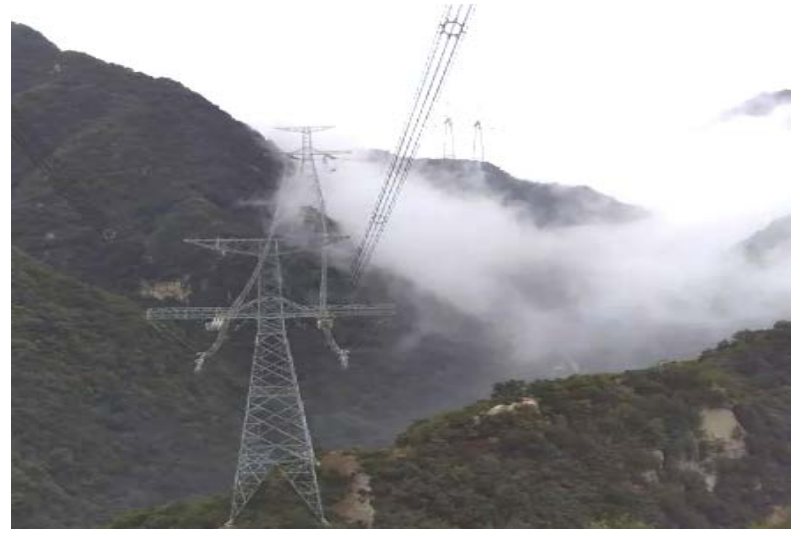

Fig.1. UHV transmission line in plateau areas

\section{Selection of Meteorological Classification Factors in Plateau}

According to the impact of the meteorological conditions of the plateau climate environment on the power supply mode of the online condition monitoring device of the transmission line in the plateau area, the main classification factors of the meteorological conditions of the climate environment of the plateau area include average sunshine, environmental wind speed, and environmental temperature. Combined with the distribution of the plateau area and the characteristics of typical climate and environment in China, the plateau area is divided into the Qinghai-Tibet Plateau, the Yungui Plateau, the Inner Mongolia Plateau and the Loess Plateau.

In these plateaus some typical meteorological factors exist naturally in its region. For example, in Qinghai-Tibet Plateau region: strong solar radiation, long sunshine duration, total annual solar radiation $140 \sim 180 \mathrm{kcal} / \mathrm{cm}^{2}$, total annual sunshine duration about $2500 \sim 3200 \mathrm{~h}$, low temperature, the highest average temperature at $20^{\circ} \mathrm{Cand}$ the lowest average temperature at $-6^{\circ} \mathrm{C}$, and the annually average wind speed about $1 \sim 3 \mathrm{~m} / \mathrm{s}$; In the Loess Plateau area: the average maximum temperature at $28^{\circ} \mathrm{C}$, the minimum average temperature at $3{ }^{\circ} \mathrm{C}$, the characteristics of severe winter and warm summer, the annual average wind speed about $3 \sim 5 \mathrm{~m} / \mathrm{s}$, and annually total sunshine hours about 2800 3200h; In the Yunnan-Guizhou Plateau area: the average maximum temperature at $24^{\circ} \mathrm{C}$ and the average lowest temperature at $5^{\circ} \mathrm{C}$, and less sunshine hours; In Inner Mongolia Plateau: one of the windy areas in China, the annual average wind speed about $7 \sim 9 \mathrm{~m} / \mathrm{s}$. the strong winds above grade 8 about 50 90 days, sufficient sunshine, the highest average temperature at $24{ }^{\circ} \mathrm{C}$, and the lowest average temperature at $-3^{\circ} \mathrm{C}$.

Based on the meteorological characteristics of the above four high plateau areas, large-scale quantitative or coarse qualitative descriptions are adopted, which is difficult to decide the right selection of the power supply source of the transmission line condition monitoring device. In addition, the above-mentioned areas have a wide classification range and insufficient data precision. There may be very similar or very different meteorological areas in or between different regions, which is largely related to the selection and use of meteorological conditions. It is extremely difficult to decide the meteorological conditions judgment and application selection. Considering that the power supply method of the condition monitoring device depends more on the local average sunshine and environmental wind speed and meteorological conditions, therefore, both of them are 
selected as the meteorological classification factors of the regional meteorological classification for the meteorological information of power supply source selection in plateau aera.

\section{3. meteorological data of typical plateau areas in China}

Here taking the meteorological data of 60 plateau cities as an example for the meteorological classification, the average sunshine $(\mathrm{h})$ and average wind speed $(\mathrm{m} / \mathrm{s})$ are shown in table $\mathrm{I}$.

TABle I. PlateAu ClimAtE ENVIRONMENTAL DATA

\begin{tabular}{|c|c|c|}
\hline Plateau regions & $\begin{array}{l}\text { Sunshine } \\
\text { (h) }\end{array}$ & $\begin{array}{c}\text { Wind speed } \\
(\mathbf{m} / \mathbf{s})\end{array}$ \\
\hline Tibet Lhasa & 2800 & 4 \\
\hline Tibet Linzhi & 1400 & 4 \\
\hline TibetShannan & 2500 & 5 \\
\hline ,Tibet Qamdo & 1800 & 4 \\
\hline Tibet Medog & 2800 & 4.7 \\
\hline Tibet Saga & 2700 & 4.5 \\
\hline Tibet Puland & 2800 & 5 \\
\hline Tibet Namco & 2500 & 4.5 \\
\hline InnerMongolia Hohhot & 3500 & 7.5 \\
\hline InnerMongolia Erenhot & 3600 & 8.5 \\
\hline Inner Mongolia Baotou & 3700 & 8 \\
\hline InnerMongolia Jining & 3500 & 7 \\
\hline InnerMongolia Linhe & 3300 & 8 \\
\hline InnerMongolia Dongsheng & 3300 & 8 \\
\hline InnerMongolia Duolun & 3500 & 9 \\
\hline NingxiaYinchuan & 2600 & 7 \\
\hline Ningxia Xiji & 2500 & 6.5 \\
\hline Ningxia Zhongwei & 2400 & 7.0 \\
\hline Ningxia Yanchi & 2600 & 6.5 \\
\hline Ningxia Guyuan & 2400 & 7.5 \\
\hline Shanxi Datong & 2400 & 5 \\
\hline Shanxi Yushe & 2600 & 4.5 \\
\hline Shanxi Wuzhai & 2800 & 5.5 \\
\hline Shaanxi Yulin & 2500 & 5 \\
\hline Shanxi Yan'an, & 2600 & 6 \\
\hline Yunnan Kunming & 1400 & 2.5 \\
\hline Yunnan Deqin & 1500 & 3 \\
\hline Yunnan Zhaotong & 1400 & 2.5 \\
\hline Yunnan Lijiang & 1300 & 3 \\
\hline YunnanTengchong & 1400 & 2 \\
\hline Yunnan Dali & 1500 & 3 \\
\hline Yunnan Yuxi & 1200 & 2.5 \\
\hline Yunnan Yiliang & 1300 & 3 \\
\hline Yunnan Chuxiong & 1400 & 3.5 \\
\hline Yunnan Qujing & 1500 & 3 \\
\hline
\end{tabular}




\begin{tabular}{|l|c|c|}
\hline Guizhou Guiyang & 1200 & 2 \\
\hline Guizhou Bijie & 1300 & 3 \\
\hline Guizhou Xishui & 1000 & 2.5 \\
\hline Guizhou Anshun & 1100 & 3.5 \\
\hline Guizhou Xingren & 1300 & 2.5 \\
\hline Guizhou Duyun & 1159 & 3.1 \\
\hline Gansu Lanzhou & 2600 & 3.4 \\
\hline Gansu Jiuquan, & 2700 & 6.5 \\
\hline Gansu Wuwei, & 2400 & 7 \\
\hline Gansu Pingliang, & 2500 & 7.5 \\
\hline Gansu Tianshui & 2700 & 5.5 \\
\hline Gansu Yumen & 2400 & 6.5 \\
\hline Gansu Dunhuang, & 2600 & 7.0 \\
\hline Qinghai Xining & 3000 & 5.5 \\
\hline Qinghai Qilian & 3500 & 6.5 \\
\hline Qinghai Golmud & 3400 & 8 \\
\hline Qinghai Delingha & 3200 & 8.5 \\
\hline Qinghai Dulan & 3300 & 8.5 \\
\hline Qinghai Zeku & 3200 & 3 \\
\hline Sichuan Ya'an & 1000 & 3 \\
\hline SichuanGuangyuan & 1200 & 4.5 \\
\hline Sichuan Ganzi & 1500 & 4.2 \\
\hline Sichuan Aba & 1800 & 5 \\
\hline Sichuan Liangshan & 3000 & 4 \\
\hline Sichuan Panzhihua & 2800 & \\
\hline & & 8 \\
\hline
\end{tabular}

\section{K-mediods Cluster algorithm and data normalization}

There are many ways to implement clustering analysis algorithms, among which K-means algorithm and K-Mediods are commonly used [6-7]. In fact, K-Mediods is basically similar to the Kmeans algorithm. Their difference is that Kmeans selects the cluster center to represent cluster clusters, and K-Mediods selects objects near the cluster center to represent cluster clusters. In the case of outliers, the robustness (stability) of K-Mediods is better [9].

The basic idea of K-mediods clustering algorithm is to select the most central object in the cluster, and try to give $\mathrm{k}$ partitions to $\mathrm{n}$ objects so that the representative object is also become the center point, and other objects are called non-representative objects. The K-mediods clustering algorithm needs to calculate the dissimilarity between all unselected objects and selected objects as the basis for grouping. Under normal circumstances, the data object is digital and the Manhattan distance is used. The distance $d$ is expressed as

$$
d(i, j)=\left|x_{i 1}-x_{j 1}\right|+\left|x_{i 2}-x_{j 2}\right|+\ldots+\left|x_{i n}-x_{j n}\right| \quad(1) \quad \text { here } \quad i=\left(x_{i 1}, x_{i 2}, \cdots, x_{i n}\right),
$$

$j=\left(x_{j 1}, x_{j 2}, \cdots, x_{j n}\right)$ represents two n-dimensional data objects.

K-mediods algorithm is described as

(1) First of all, randomly select a set of cluster samples as the center point set. 
(2) Each central point corresponds to a cluster.

(3) Calculate the distance between each sample point and each center point (such as Manhattan distance), and put the sample point into the cluster with the shortest distance from the center point.

(4) In each cluster calculate the point of the smallest absolute distance from each sample point in the cluster as the new center point.

(5) If the new center point set is the same as the original center point set, the algorithm terminates; if the new center point set is not exactly the same as the original center point set, return to (2).

Generally, the measured data should be pre-normalized to eliminate the abnormal clustering analysis results caused by the difference from the measured data dimension and the range of values. The normalized maximum-minimum (MAX-MIN) conversion is defined as follows

$$
x^{*}=\frac{x-\min (x)}{\max (x)-\min (x)}
$$

Here $\max (x)$ is the biggest measured data and $\min (x)$ is the smallest measured data, the $\max (x)-\min (x)$ is of the extreme difference the measured data.

\section{Classification realization of environmental meteorological factors in plateau}

After pre-processing the data in table I, the K-mediods fern analysis algorithm was used for cluster analysis, and its clustering results were shown in Fig.2 and Fig.3, respectively. In Fig.3, 60 meteorological data were divided into 4 categories, and the classification information was shown in this figure: category 1 contains 15 data, category 2 contains 22 data, category 3 contains 12 data, and category 4 contains 11 data. In Fig.3, 4 classification data were all distributed near their central points, and the classification groups were ideal.

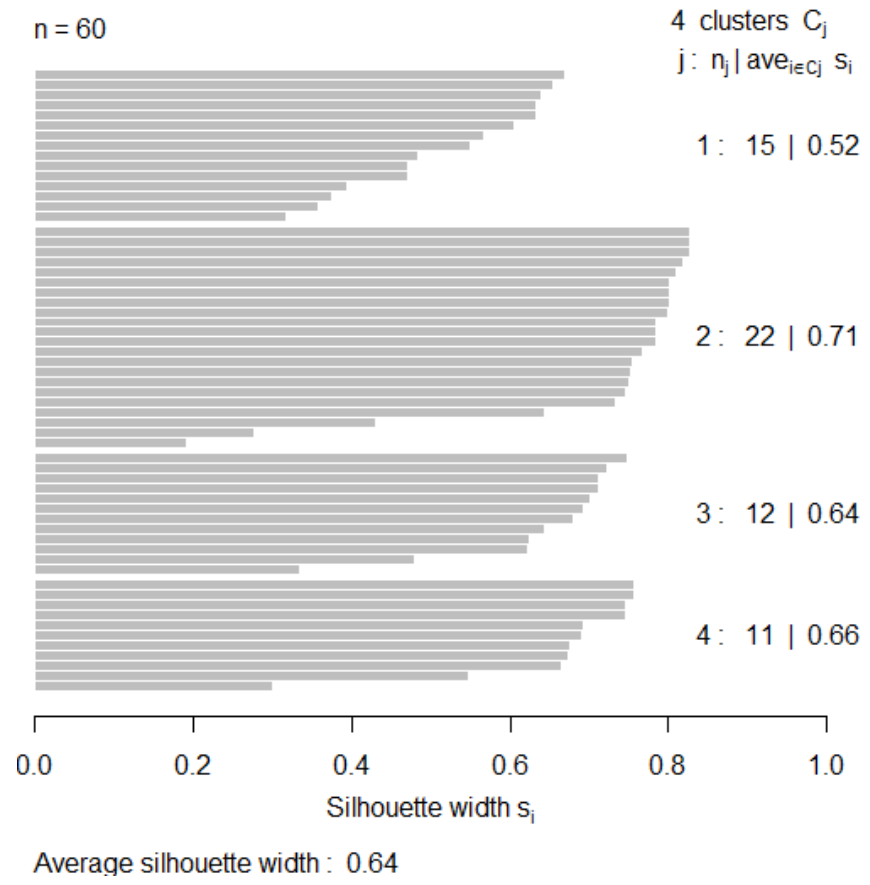

Fig.2 Silhouette of data clustering 
According to the classification result in Fig.3, the meteorological data in table I was rearranged as shown in table II. In this way, all the meteorological data could be used to engineering application according to the environmental meteorological classification.

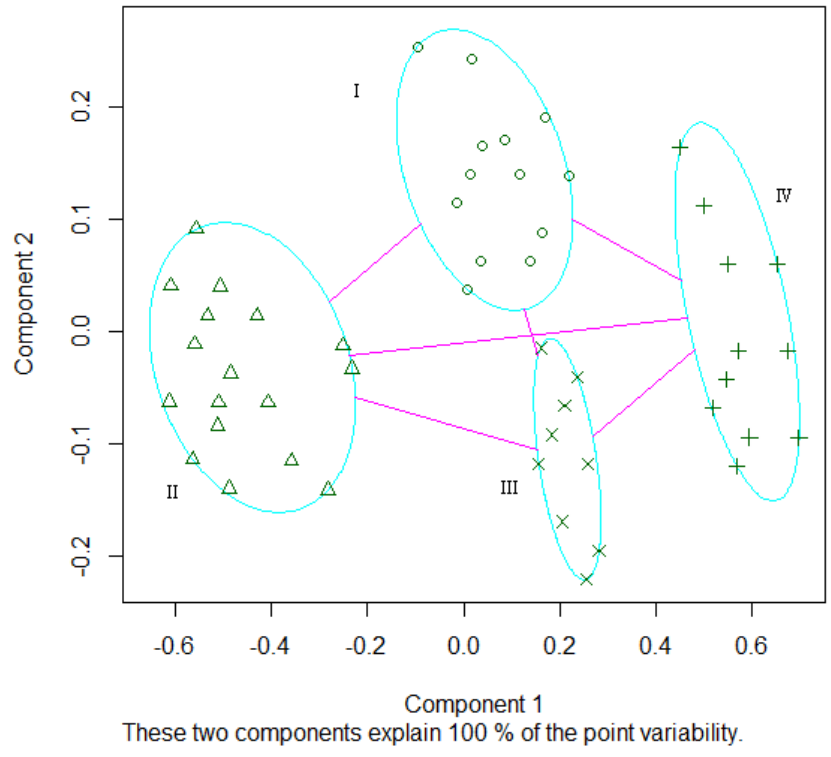

Fig.3 Silhouette of data clustering

TABLE II. CLUSTER REARRANGEMENT OF CLIMATE ENVIRONMENTAL DATA

\begin{tabular}{|l|c|c|c|}
\hline Plateau regions & $\begin{array}{c}\text { Sunshine } \\
(\mathbf{h})\end{array}$ & $\begin{array}{c}\text { Wind speed } \\
(\mathbf{m} / \mathbf{s})\end{array}$ & \multicolumn{2}{|c|}{ Clusters } \\
\hline Tibet Lhasa & 2800 & 4 & 1 \\
\hline TibetShannan & 2500 & 5 & 1 \\
\hline Tibet Medog & 2800 & 4.7 & 1 \\
\hline Tibet Saga & 2700 & 4.5 & 1 \\
\hline Tibet Puland & 2800 & 5 & 1 \\
\hline Tibet Namco & 2500 & 4.5 & 1 \\
\hline Shanxi Datong & 2400 & 5 & 1 \\
\hline Shanxi Yushe & 2600 & 4.5 & 1 \\
\hline Shanxi Wuzhai & 2800 & 5.5 & 1 \\
\hline Shaanxi Yulin & 2500 & 5 & 1 \\
\hline Gansu Lanzhou & 2600 & 3.4 & 1 \\
\hline Gansu Tianshui & 2700 & 5.5 & 1 \\
\hline Qinghai Xining & 3000 & 5.5 & 1 \\
\hline Sichuan Liangshan & 3000 & 5 & 1 \\
\hline Sichuan Panzhihua & 2800 & 4 & 1 \\
\hline Tibet Linzhi & 1400 & 4 & 2 \\
\hline Tibet Qamdo & 1800 & 4 & 2 \\
\hline Yunnan Kunming & 1400 & 2.5 & 2 \\
\hline Yunnan Deqin & 1500 & 3 & 2 \\
\hline Yunnan Zhaotong & 1400 & 2.5 & 2 \\
\hline Yunnan Lijiang & 1300 & 3 & 2 \\
\hline
\end{tabular}




\begin{tabular}{|c|c|c|c|c|}
\hline YunnanTengchong & 1400 & 2 & 2 & \\
\hline Yunnan Dali & 1500 & 3 & 2 & \\
\hline Yunnan Yuxi & 1200 & 2.5 & 2 & \\
\hline Yunnan Yiliang & 1300 & 3 & 2 & \\
\hline Yunnan Chuxiong & 1400 & 3.5 & 2 & \\
\hline Yunnan Qujing & 1500 & 3 & 2 & \\
\hline Guizhou Guiyang & 1200 & 2 & 2 & \\
\hline Guizhou Bijie & 1300 & 3 & 2 & \\
\hline Guizhou Xishui & 1000 & 2.5 & 2 & \\
\hline Guizhou Anshun & 1100 & 3.5 & 2 & \\
\hline Guizhou Xingren & 1300 & 2.5 & 2 & \\
\hline Guizhou Duyun & 1159 & 3.1 & 2 & \\
\hline Sichuan Ya'an & 1000 & 3 & 2 & \\
\hline SichuanGuangyuan & 1200 & 3 & 2 & \\
\hline Sichuan Ganzi & 1500 & 4.5 & 2 & \\
\hline Sichuan Aba & 1800 & 4.2 & 2 & \\
\hline InnerMongolia Hohhot & 3500 & 7.5 & 3 & \multirow{12}{*}{ III } \\
\hline InnerMongolia Erenhot & 3600 & 8.5 & 3 & \\
\hline Inner Mongolia Baotou & 3700 & 8 & 3 & \\
\hline InnerMongolia Jining & 3500 & 7 & 3 & \\
\hline InnerMongolia Linhe & 3300 & 8 & 3 & \\
\hline InnerMongolia Dongsheng & 3300 & 8 & 3 & \\
\hline InnerMongolia Duolun & 3500 & 9 & 3 & \\
\hline Qinghai Qilian & 3500 & 6.5 & 3 & \\
\hline Qinghai Golmud & 3400 & 8 & 3 & \\
\hline Qinghai Delingha & 3200 & 8 & 3 & \\
\hline Qinghai Dulan & 3300 & 8.5 & 3 & \\
\hline Qinghai Zeku & 3200 & 8.5 & 3 & \\
\hline NingxiaYinchuan & 2600 & 7 & 4 & \multirow{11}{*}{ IV } \\
\hline Ningxia Xiji & 2500 & 6.5 & 4 & \\
\hline Ningxia Zhongwei & 2400 & 7 & 4 & \\
\hline Ningxia Yanchi & 2600 & 6.5 & 4 & \\
\hline Ningxia Guyuan & 2400 & 7.5 & 4 & \\
\hline Shanxi Yan'an, & 2600 & 6 & 4 & \\
\hline Gansu Jiuquan, & 2700 & 6.5 & 4 & \\
\hline Gansu Wuwei, & 2400 & 7 & 4 & \\
\hline Gansu Pingliang, & 2500 & 7.5 & 4 & \\
\hline Gansu Yumen & 2400 & 6.5 & 4 & \\
\hline Gansu Dunhuang, & 2600 & 7 & 4 & \\
\hline
\end{tabular}

According to the environmental meteorological factors in Table 2, four types of environmental meteorological areas were classified and defined a new weather description for the meteorological characteristics of the plateau area, all of which is convenient for the engineering applications of condition monitoring power supply. 
The new definition descriptions of environmental meteorological areas are as follows: strong solar and wind energy area, medium solar and strong wind energy area, medium solar and wind energy area, and weak solar and wind energy area. These meteorological characteristics areas corresponding to the clustering groups in table II are shown in table III.

TABLE III. Cluster VS. MeteOROLOGICAL CHARACTERISTICS

\begin{tabular}{|c|l|}
\hline Cluster & Meteorological characteristics \\
\hline I & medium solar and wind energy \\
\hline II & weak solar and wind energy \\
\hline III & strong solar and wind energy \\
\hline IV & medium solar and strong wind energy \\
\hline
\end{tabular}

\section{Conclusions}

After the analysis of the potential constraints affecting the stability and reliable power supply of the condition monitoring device in the plateau area, the main influencing aspects of the environmental meteorological conditions in the plateau area were obtained, and then the environmental meteorological classification factors were effectively proposed and the plateau area classification based on the meteorological conditions were realized, and the comparative analysis showed that the classification results were reasonable and effective.

Based on the meteorological characteristics of the plateau environment area, each corresponding meteorological area were defined according to the clustering groups, and so the basic technical requirements for the selection of the power supply mode of the condition monitoring device of the transmission line could be realized well, which were convenient for the engineering application of plateau meteorological environment.

\section{Acknowledgment}

We are greatly indebted to people who helped us a lot. This work was funded by the project "Study on the Energy Supply and Signal Transmission Technology of Transmission Line Condition Monitoring Device in Plateau Area (521997170016)” from the State Grid Sichuan Electric Power Company.

\section{References}

[1] LIU lei, TIAN Feng, LI Min, ZENG Rong,YU Zhangqing,Prediction of Electromagnetic Environment on High Altitude UHVDC Transmission Lines with Physical Factors,Southern Power Systerm Technology, 2016,vol.10(9),pp.43-48.

[2] LIU lei, LI Min, LI Ruihai. Influence of Meteorological Parameters on Electromagnetic Environment of UHVDC Transmission Line Under High Altitude Condition, High Voltage Engineering,2012, vol.38(12), pp.3177-3181.

[3] CHEN Zhonghua,YU Rongjiang,HU Chengang, CHEN Pan,GAO Zhenyu, Energy Management Strategy of Wind-solar Hybrid Power System for Transmission Line On-line Monitoring Equipment, Power \& Energy, 2017,vol.38(4),pp.387-390. 
[4] XIAO Junming, WEI Xuehui, LI Yanbin, ZHOU Qian, CHEN Xiang,Optimization design of distributed generation in stand-alone wind-solar hybrid system,Electrical Measurement \&Instrumentation, 2016, vol.53 (6) pp.118-123.

[5] ZHI Lixiang,LIU Zhe,ZHANG Lidong,LU Wenqiang,Research and application of power supply for high voltage transmission lines online monitoring, Chinese Journal of Power Sources, 2015,vol.39(2),pp.413-415.

[6] Devesh Kumar Srivastava ,Ravinder Yadav ,Gaurav Agrwal, Map reduce programming model for parallel K-mediod algorithm on hadoop cluster, 2017 7th International Conference on Communication Systems and Network Technologies (CSNT), India,2017.

[7] Xinran Zhang, David J. Hill, Clustering of Uncertain Load Model Parameters with K-medoids Algorithm, 2018 IEEE Power \& Energy Society General Meeting (PESGM), Portland,2018.

[8] Shen kaili Shen,Wei Liu, Tao Zhang, Load Pattern Recognition and Prediction Based on DTW K-Mediods Clustering and Markov Model, 2019 IEEE International Conference on Energy Internet (ICEI),2019.

[9] Romana Riyaz ,Mohd Arif Wani, Comparative study of cluster validity techniques using Kmediod algorithm, 2015 2nd International Conference on Computing for Sustainable Global Development (INDIACom), India,2015. 